\title{
A Concentration Phenomenon for p-Laplacian Equation
}

\author{
Yansheng Zhong \\ Department of Mathematics, Fujian Normal University, Fuzhou 350117, China \\ Correspondence should be addressed to Yansheng Zhong; zhyansheng08@163.com
}

Received 27 April 2014; Accepted 8 July 2014; Published 20 July 2014

Academic Editor: Chao Yan

Copyright (C) 2014 Yansheng Zhong. This is an open access article distributed under the Creative Commons Attribution License, which permits unrestricted use, distribution, and reproduction in any medium, provided the original work is properly cited.

It is proved that if the bounded function of coefficient $Q_{n}$ in the following equation $-\operatorname{div}\left\{|\nabla u|^{p-2} \nabla u\right\}+V(x)|u|^{p-2} u=$ $Q_{n}(x)|u|^{q-2} u, u(x)=0$ as $x \in \partial \Omega . u(x) \longrightarrow 0$ as $|x| \longrightarrow \infty$ is positive in a region contained in $\Omega$ and negative outside the region, the sets $\left\{Q_{n}>0\right\}$ shrink to a point $x_{0} \in \Omega$ as $n \rightarrow \infty$, and then the sequence $u_{n}$ generated by the nontrivial solution of the same equation, corresponding to $Q_{n}$, will concentrate at $x_{0}$ with respect to $W_{0}^{1, p}(\Omega)$ and certain $L^{s}(\Omega)$-norms. In addition, if the sets $\left\{Q_{n}>0\right\}$ shrink to finite points, the corresponding ground states $\left\{u_{n}\right\}$ only concentrate at one of these points. These conclusions extend the results proved in the work of Ackermann and Szulkin (2013) for case $p=2$.

\section{Introduction}

We study a new concentration phenomenon for the following p-Laplacian equations:

$$
\begin{aligned}
& -\operatorname{div}\left\{|\nabla u|^{p-2} \nabla u\right\}+V(x)|u|^{p-2} u=Q_{n}(x)|u|^{q-2} u, \\
& u(x)=0 \quad \text { as } x \in \partial \Omega . \\
& u(x) \longrightarrow 0 \text { as }|x| \longrightarrow \infty,
\end{aligned}
$$

where $\Omega \subset \mathbb{R}^{N}$ is a smooth domain and $V \geqslant 0\left(\in L^{\infty}(\Omega)\right)$, and $p<q<p^{*}$, where $p^{*}:=N p /(N-p)$ if $N \geqslant p$ and $p^{*}:=\infty$ if $N<p$. If $\Omega$ is unbounded, we assume additionally that $\sigma\left(-\operatorname{div}\left(|\nabla \cdot|^{p-2} \nabla \cdot\right)+V|\cdot|^{p-2} \cdot\right) \subset(0, \infty)$.

And an assumption of $Q_{n}$ is as follows.

(*) The set $\left\{x \mid Q_{n}(x)>0\right\}$ contained in the neighborhood of zero has positive measure, and $\left|Q_{n}\right|_{L^{\infty}(\Omega)} \leqslant C$ with the constant $C$ is independent of $n$. Moreover, for each $\epsilon>0$ there exist constants $\delta_{\epsilon}(>0)$ and $N_{\epsilon}$ such that $Q_{n} \leqslant-\delta_{\epsilon}$ whenever $x \notin B_{\epsilon}(0)$ and $n \geqslant N_{\epsilon}$.

As it is known, $u \equiv 0$ is the only solution to (1) if $Q_{n}(x) \leqslant 0$ for all $x \in \Omega$. In addition, if $Q_{n}(x)>0$ is based on a bounded set of positive measures, it is clear that there exists a solution $u \neq \equiv$ (see Theorem 1 ). Hence, without loss of generality, we assume that $0 \in \Omega$ and let $Q=Q_{n}$ be such that $Q_{n}>0$ on the ball $B_{1 / n}(0)$ and $Q_{n}<0$ on $\Omega \backslash B_{2 / n}(0)$ and $u_{n} \not \equiv 0$ are the solutions to (1) associated with $Q_{n}(x)$.
Accordingly, the question is what happens to $u_{n}$ as $n \rightarrow \infty$. Furthermore, this phenomenon can be found in physics. For instance, considering the materials separately from $Q$ positive or negative (see [1]), it corresponds to investigating the existence of bright $(Q>0)$ or dark $(Q<0)$ solitons.

Equations of these types have been studied extensively in many monographs and lectures (e.g., $[2-10]$ for $p=2$, [1118] for general $p$ ). In [2], Byeon and Wang considered the standing wave solutions $\psi(x, t) \equiv \exp (-i E t / \hbar) v(x)$ for the nonlinear Schrödinger equation:

$$
i \hbar \frac{\partial \psi}{\partial t}+\frac{\hbar^{2}}{2} \Delta \psi-V(x) \psi+|\psi|^{p-1} \psi=0, \quad(t, x) \in \mathbb{R} \times \mathbb{R}^{N} .
$$

Thus, they needed only to discuss the function $v$ which satisfies

$$
\frac{\hbar^{2}}{2} \Delta v-(V(x)-E) v+|v|^{p-1} v=0, \quad x \in \mathbb{R}^{N},
$$

and rewrote it in the following form:

$$
\begin{gathered}
\epsilon^{2} \Delta v-V(x) v+v^{p}=0, \quad v>0, x \in \mathbb{R}^{N} \\
\lim _{|x| \rightarrow 0} v(x)=0 .
\end{gathered}
$$


By a rescaling, it is transformed to

$$
\begin{gathered}
\Delta u-V(\epsilon x) u+u^{p}=0, \quad u>0, x \in \mathbb{R}^{N} \\
\lim _{|x| \rightarrow 0} u(x)=0 .
\end{gathered}
$$

Let the zero set $\mathscr{Z} \triangleq\left\{x \in \mathbb{R}^{N} \mid V(x)=0\right\}$ and $A$ be an isolated component of $\mathscr{Z}$, and they distinguished three cases of $A$ to prove the concentration as $\epsilon \rightarrow 0$. And then, in [3] by replacing $v^{p}$ with a fairly general class nonlinearity $f(v)$, they also obtained the concentration. Furthermore, in [4], Byeon and Jeanjean gave the almost optimal condition on $f$ for the concentration. Recently, in [19], different from above with the linearity term $V(\epsilon x) u$, Ackermann and Szulkin considered the concentration phenomenon in the nonlinearity; that is, $-\Delta u+V(x) u=Q_{n}(x)|u|^{p-2} u$. In contrast, by following the similar strategy as in [19], we first show that the concentration phenomenon also occurs in the general p-Laplacian equation. It seems that this concentration phenomenon was unknown earlier, but to some extent, it answers the question mentioned above.

This paper is organized as follows. In Section 2, we prove that the solutions $\left\{u_{n}\right\}$ to (1) concentrate at the origin in the $W_{0}^{1, p}(\Omega)$ and the $L^{q}$-norm; in Section 3, concentration in the $L^{s}$-norms for different $s$ is considered and Section 4 shows that the ground states only concentrate at one of these points when $Q_{n}$ is positive in a neighbourhood of a finite number of points.

\section{Concentration in the $W_{0}^{1, p}(\Omega)$ and $L^{q}(\Omega)$}

We begin with some notations.

Let $E:=W_{0}^{1, p}(\Omega)$ and

$$
\|u\|:=\left(\int_{\Omega}\left(|\nabla u|^{p}+V|u|^{p}\right) d x\right)^{1 / p}
$$

is an equivalent norm in $E$ (due to $\sigma\left(-\operatorname{div}\left(|\nabla \cdot|^{p-2} \nabla \cdot\right)+\right.$ $\left.\left.V|\cdot|^{p-2} \cdot\right) \subset(0, \infty)\right)$. Set

$$
|u|_{s, A}:=\left(\int_{A}|u|^{s} d x\right)^{1 / s}
$$

$|u|_{\infty, A}=\operatorname{esssup}_{A}|u|$, and we abbreviate $|u|_{s, A}$ to $|u|_{s}$ sometimes. Moreover,

$$
B_{r}(a):=\left\{x \in \mathbb{R}^{n}:|x-a|<r\right\}
$$

denotes a ball.

Here we offer the existence result for (1).

Theorem 1. Suppose that $Q_{n}$ satisfies the assumption (*) above and $q \in\left(p, p^{*}\right)$; then for all sufficiently large $n$, there is a positive ground state solution $u_{n} \in E$ to problem (1). Moreover, there exists a constant $\alpha>0$ independent of $n$, such that $\left\|u_{n}\right\| \geqslant \alpha$.

Proof. As in [19], let $J_{n}(v)=\int_{\Omega} Q_{n}|v|^{q} d x$ and

$$
s_{n}:=\inf _{J_{n}(v)>0} \frac{\|v\|^{p}}{\left|J_{n}(v)\right|^{p / q}}=\inf _{J_{n}(v)>0} \frac{\int_{\Omega}|\nabla v|^{p}+V|v|^{p} d x}{\left(\int_{\Omega} Q_{n}|v|^{q} d x\right)^{p / q}} .
$$

Suppose that $\left(v_{k}\right)$ is a minimizing sequence for $s_{n}$, normalized by $J_{n}\left(v_{k}\right)=1$; then $\left\|v_{k}\right\|$ is bounded. Hence, $v_{n} \rightarrow v$ in $E$ and $v_{k}(x) \rightarrow v(x)$ a.e. in $\Omega$ (by choosing a subsequence). Note that $Q_{n}<0$ on $|x|>1$ for $n$ large. The Rellich-Kondrachov Theorem and Fatous's Lemma say that

$$
\begin{aligned}
s_{n} & =\lim _{k \rightarrow \infty}\left\|v_{k}\right\|^{p} \\
& =\lim _{k \rightarrow \infty} \frac{\left\|v_{k}\right\|^{p}}{\left(\int_{|x|<1} Q_{n}\left|v_{k}\right|^{q} d x+\int_{|x|>1} Q_{n}\left|v_{k}\right|^{q} d x\right)^{p / q}} \\
& \geqslant \frac{\|v\|^{p}}{J_{n}(v)^{p / q}} \geqslant s_{n} .
\end{aligned}
$$

Thus $v$ is a minimizer.

And then, the lagrange multiple rule implies that $u_{n}=$ $c_{n} v_{n}$ is a solution to (1) for some appropriate constant $c_{n}>0$. Moreover, since $v_{n}$ may be replaced by $\left|v_{n}\right|, v_{n} \geqslant 0$ (and hence $\left.u_{n} \geqslant 0\right)$. To show that $u_{n}>0$, we note that $u_{n}$ satisfies

$$
\begin{aligned}
& -\operatorname{div}\left(|\nabla v|^{p-2} \nabla v\right)+\left(V(x) u_{n}^{p-2}+Q_{n}^{-}(x) u_{n}(x)^{q-2}\right) v \\
& =Q_{n}^{+}(x) u_{n}(x)^{q-1} \geqslant 0,
\end{aligned}
$$

where $Q_{n}^{ \pm}:=\max \left\{ \pm Q_{n}(x), 0\right\}$. Since $V(x) u_{n}^{p-2}+Q_{n}^{-}(x) u_{n}$ $(x)^{q-2} \geqslant 0$, it follows from the strong maximum principle (see $[20,21])$ that $u_{n}>0$.

If $u_{n} \neq 0$ is a solution to (1), then, via multiplying the equation by $u_{n}$, integrating by parts, and using the Sobolev inequality, one deduces that

$$
\left\|u_{n}\right\|^{p}=\int_{\Omega} Q_{n}\left|u_{n}\right|^{q} d x \leqslant c_{1}\left|u_{n}\right|_{q}^{q} \leqslant c_{2}\left\|u_{n}\right\|^{q}
$$

hence, $\left\|u_{n}\right\| \geqslant \alpha$ for some $\alpha>0$ and all large $n$.

The next step is to consider the property of the nontrivial solution $\left\{u_{n}\right\}$ to $(1)$ and $w_{n}:=u_{n} /\left\|u_{n}\right\|$.

Lemma 2. Consider

$$
\left\|u_{n}\right\| \longrightarrow \infty \text { as } n \longrightarrow \infty \text {. }
$$

Proof. We present an abridged version of the proof highlighting the main differences to that in [19]. It will be proved by contradiction. Assume $u_{n} \rightarrow u$ in $E$ and $u_{n} \rightarrow u$ in $L_{\text {loc }}^{q}(\Omega)$ after passing to a subsequence. Multiplying (1) (with $u=u_{n}$ ) by $u_{n}$, integrating by parts, and recalling that $Q_{n}<0$ for each $\epsilon>0$ and $n \geqslant N_{\epsilon}$, it holds that

$$
\begin{aligned}
\limsup _{n \rightarrow \infty}\left\|u_{n}\right\|^{p} & =\limsup _{n \rightarrow \infty} \int_{\Omega} Q_{n}\left|u_{n}\right|^{q} d x \\
& \leqslant \limsup _{n \rightarrow \infty} \int_{|x|<\epsilon} Q_{n}\left|u_{n}\right|^{q} d x \leqslant c \int_{|x|<\epsilon}|u|^{q} d x
\end{aligned}
$$


If $\epsilon \rightarrow 0, u_{n} \rightarrow 0$ in $E$. It is a contradiction to $\left\|u_{n}\right\| \geqslant$ $\alpha>0$ given in Theorem 1 .

Lemma 3. Consider

$$
w_{n} \rightarrow 0 \text { in } E \text { as } n \longrightarrow \infty
$$

Proof. We prove it by contradiction as well. We may assume that $w_{n} \rightarrow w(\neq \equiv 0)$ in E. Multiplying (1) (with $u=u_{n}$ ) by $u_{n} /\left\|u_{n}\right\|^{p}$ yields that

$$
1=\left\|w_{n}\right\|^{p}=\left\|u_{n}\right\|^{q-p} \int_{\Omega} Q_{n}\left|w_{n}\right|^{q} d x .
$$

Due to Lemma 2 with $q>p, \int_{\Omega} Q_{n}\left|w_{n}\right|^{q} \rightarrow 0$.

On the other hand, we have for $0<\epsilon<\epsilon_{1}$

$$
\begin{aligned}
0 & =\lim _{n \rightarrow \infty} \int_{\Omega} Q_{n}\left|w_{n}\right|^{q} d x \\
& =\lim _{n \rightarrow \infty}\left(\int_{|x|<\epsilon} Q_{n}\left|w_{n}\right|^{q} d x+\int_{|x|>\epsilon} Q_{n}\left|w_{n}\right|^{q} d x\right) \\
& \leqslant \lim _{n \rightarrow \infty}\left(\int_{|x|<\epsilon} Q_{n}\left|w_{n}\right|^{q} d x+\int_{|x|>\epsilon_{1}} Q_{n}\left|w_{n}\right|^{q} d x\right) \\
& \leqslant c \int_{|x|<\epsilon}\left|w_{n}\right|^{q} d x-\delta_{\epsilon_{1}} \int_{|x|>\epsilon_{1}}\left|w_{n}\right|^{q} d x .
\end{aligned}
$$

We may choose small $\epsilon_{1}$ such that the second integral on the right-hand side above is positive as $w \not \equiv 0$. Then we get the contradiction as $\epsilon \rightarrow 0$.

In the sequel, we study concentration of $\left\{u_{n}\right\}$ as $n \rightarrow \infty$. Let $\epsilon>0$ be given and $\chi \in C^{\infty}(\Omega,[0,1])$ be such that $\chi(x)=$ 0 for $x \in B_{\epsilon / 2}(0)$ and $\chi(x)=1$ for $x \notin B_{\epsilon}(0)$.

Multiplying (1) (with $u=u_{n}$ ) by $\chi u_{n}$ we obtain

$$
\int_{\Omega}\left(\left|\nabla u_{n}\right|^{p-2} \nabla u_{n} \cdot \nabla\left(\chi u_{n}\right)+\chi V u_{n}^{p}\right) d x=\int_{\Omega} \chi Q_{n}\left|u_{n}\right|^{q} d x,
$$

namely,

$$
\begin{gathered}
\int_{\Omega} \chi\left(\left|\nabla u_{n}\right|^{p}+V u_{n}^{p}\right) d x-\int_{\Omega} \chi Q_{n}\left|u_{n}\right|^{q} d x \\
=-\int_{\Omega}\left|\nabla u_{n}\right|^{p-2} \nabla u_{n} \cdot \nabla \chi \cdot u_{n} d x .
\end{gathered}
$$

Given $\epsilon>0$, we have $Q_{n} \leqslant-\delta_{\epsilon}$ on $\operatorname{supp} \chi$, provided that $n$ is large enough. Hence for all such $n$,

$$
\begin{aligned}
0 & \leqslant \int_{\Omega \backslash B_{\epsilon}(0)}\left(\left|\nabla u_{n}\right|^{p}+V u_{n}^{p}\right) d x+\delta_{\epsilon} \int_{\Omega \backslash B_{\epsilon}(0)}\left|u_{n}\right|^{q} d x \\
& \leqslant \int_{\Omega} \chi\left(\left|\nabla u_{n}\right|^{p}+V u_{n}^{p}\right) d x-\int_{\Omega} \chi Q_{n}\left|u_{n}\right|^{q} d x \\
& =-\int_{\Omega}\left|\nabla u_{n}\right|^{p-2} \nabla u_{n} \cdot \nabla \chi \cdot u_{n} d x \\
& \leqslant d_{\epsilon} \int_{B_{\epsilon}(0) \backslash B_{\epsilon / 2}(0)}\left|u_{n}\right|\left|\nabla u_{n}\right|^{p-1} d x,
\end{aligned}
$$

where $d_{\epsilon}$ is a constant independent of $n$. Since $w_{n}=u_{n} /$ $\left\|u_{n}\right\| \rightarrow 0$ in $L_{\text {loc }}^{p}(\Omega)$ according to Lemma 3 , it follows from Hölder inequality that

$$
\int_{B_{\epsilon}(0) \backslash B_{\varepsilon / 2}(0)}\left|w_{n}\right|\left|\nabla w_{n}\right|^{p-1} d x \longrightarrow 0 .
$$

So (20) implies

$$
\int_{\Omega \backslash B_{\epsilon}(0)}\left(\left|\nabla w_{n}\right|^{p}+V w_{n}^{p}\right) d x+\delta_{\epsilon}\left\|u_{n}\right\|^{q-p} \int_{\Omega \backslash B_{\epsilon}(0)}\left|w_{n}\right|^{q} d x=0 .
$$

Theorem 4. Suppose that $Q_{n}$ satisfies the assumption $(*)$ and $q \in\left(p, p^{*}\right)$. Let $u_{n}$ be a nontrivial solution to (1) and put $w_{n}=$ $u_{n} /\left\|u_{n}\right\|$. Then for every $\epsilon>0$ they hold that

$$
\begin{gathered}
\lim _{n \rightarrow \infty} \int_{\Omega \backslash B_{\epsilon}(0)}\left(\left|\nabla w_{n}\right|^{p}+V w_{n}^{p}\right) d x=0, \\
\lim _{n \rightarrow \infty}\left\|u_{n}\right\|^{q-p} \int_{\Omega \backslash B_{\varepsilon}(0)}\left|w_{n}\right|^{q} d x=0 .
\end{gathered}
$$

Moreover,

$$
\begin{aligned}
& \lim _{n \rightarrow \infty} \frac{\int_{\Omega \backslash B_{\varepsilon}(0)}\left(\left|\nabla w_{n}\right|^{p}+V w_{n}^{p}\right) d x}{\int_{\Omega}\left(\left|\nabla w_{n}\right|^{p}+V w_{n}^{p}\right) d x}=0, \\
& \lim _{n \rightarrow \infty} \frac{\int_{\Omega \backslash B_{\epsilon}(0)}\left|w_{n}\right|^{q} d x}{\int_{\Omega}\left|w_{n}\right|^{q} d x}=0 .
\end{aligned}
$$

Proof. (23) and (24) can be easily obtained by (22). Note that

$$
\int_{\Omega}\left(\left|\nabla w_{n}\right|^{p}+V w_{n}^{p}\right) d x=\left\|w_{n}\right\|^{p}=1 .
$$

From (23), one concludes that

$$
\begin{aligned}
& \lim _{n \rightarrow \infty} \frac{\int_{\Omega \backslash B_{\varepsilon}(0)}\left(\left|\nabla u_{n}\right|^{p}+V u_{n}^{p}\right) d x}{\int_{\Omega}\left(\left|\nabla u_{n}\right|^{p}+V u_{n}^{p}\right) d x} \\
& =\lim _{n \rightarrow \infty} \frac{\int_{\Omega \backslash B_{\varepsilon}(0)}\left(\left|\nabla w_{n}\right|^{p}+V w_{n}^{p}\right) d x}{\int_{\Omega}\left(\left|\nabla w_{n}\right|^{p}+V w_{n}^{p}\right) d x}=0 .
\end{aligned}
$$

According to (16), we get

$$
c\left\|u_{n}\right\|^{q-p} \int_{\Omega}\left|w_{n}\right|^{q} d x \geqslant\left\|u_{n}\right\|^{q-p} \int_{\Omega} Q_{n}\left|w_{n}\right|^{q} d x=\left\|w_{n}\right\|^{p}=1 .
$$

This and (24) imply

$$
\lim _{n \rightarrow \infty} \frac{\int_{\Omega \backslash B_{\varepsilon}(0)}\left|w_{n}\right|^{q} d x}{\int_{\Omega}\left|w_{n}\right|^{q} d x}=\lim _{n \rightarrow \infty} \frac{\left\|u_{n}\right\|^{q-p} \int_{\Omega \backslash B_{\varepsilon}(0)}\left|w_{n}\right|^{q} d x}{\left\|u_{n}\right\|^{q-p} \int_{\Omega}\left|w_{n}\right|^{q} d x}=0 .
$$




\section{Concentration in the $L^{s}$-Norm}

The next is to consider the concentration in other norms.

Theorem 5. Let $u_{n}$ denote a nontrivial solution to (1) for each $n \in \mathbb{N}$. Suppose that the assumption $(*)$ holds and there exists $R, \lambda>0$ such that $V \geqslant \lambda$ whenever $x \in \Omega \backslash B_{R}(0)$, and there exists $\epsilon>0$ such that $\overline{B_{\epsilon}(0)} \subset \Omega$; then one can get that

(a) $\exists C$, for all $s \in[1, \infty], n \in \mathbb{N},\left|u_{n}\right|_{s, \Omega \backslash B_{\varepsilon}(0)} \leqslant C$;

(b) if $\delta=\delta_{\epsilon}>0$ in $(*)$ can be chosen independently of $\epsilon(>$ $0)$, then $\lim _{n \rightarrow \infty}\left|u_{n}\right|_{s, \Omega \backslash B_{\epsilon}(0)}=0$, for every $s \in[1, \infty]$;

(c) for all $s(\geqslant 1) \in(N(q-p) / p, \infty]$, one has $\lim _{n \rightarrow \infty}$ $\left|u_{n}\right|_{s}=\infty$ and

$$
\lim _{n \rightarrow \infty} \frac{\left|u_{n}\right|_{s, \Omega \backslash B_{\varepsilon}(0)}}{\left|u_{n}\right|_{s}}=0
$$

(d) if $N(q-p) / p \geqslant 1$, then for $s=N(q-p) / p$ it holds that

$$
\liminf _{n \rightarrow \infty}\left|u_{n}\right|_{s}>0
$$

If the hypotheses in (b) are satisfied, then (30) also holds for this $s$.

Proof. There is clearly a positive classical solution $w$ to the equation

$$
\begin{aligned}
& -\operatorname{div}\left(|\nabla u|^{p-2} \nabla u\right)=-\delta_{\epsilon / 2}|u|^{q-2} u, \quad x \in \mathbb{R}^{n} \backslash \overline{B_{\epsilon / 2}(0)} \\
& \lim _{|x| \rightarrow \epsilon / 2} w(x)=\infty, \quad \lim _{|x| \rightarrow \infty} w(x)=0 .
\end{aligned}
$$

In fact, by $[22,23]$, the radial solution $u_{p}(x)=u_{p}(|x|)$ satisfies the ordinary differential equation

$$
\begin{aligned}
& \left(r^{n-1}\left|u^{\prime}\right|^{p-2} u^{\prime}\right)^{\prime}=-\delta_{\epsilon / 2} r^{n-1} u^{q} \\
& u(r)=\infty \text { as } r \longrightarrow \epsilon / 2, \\
& u(r) \longrightarrow 0 \text { as } r \longrightarrow \infty .
\end{aligned}
$$

Set $z_{n}=w-u_{n}$ and

$$
\begin{aligned}
& \varphi_{n}(x):=(q-1) \int_{0}^{1}\left|s w(x)+(1-s) u_{n}(x)\right|^{q-2} d s \geqslant 0, \\
& \phi_{n}(x):=(p-1) \int_{0}^{1}\left|s w(x)+(1-s) u_{n}(x)\right|^{p-2} d s \geqslant 0,
\end{aligned}
$$

$$
\begin{aligned}
\varphi_{n} & (x) z_{n} \\
& =(q-1) \int_{0}^{1}\left|s w(x)+(1-s) u_{n}(x)\right|^{q-2}\left(w-u_{n}\right) d s \\
& =\int_{0}^{1} \frac{d}{d s}\left(\left|s w+(1-s) u_{n}\right|^{p-2}\left(s w+(1-s) u_{n}\right)\right) d s \\
& =w^{q-1}-\left|u_{n}\right|^{q-2} u_{n}, \\
\phi_{n} & (x) z_{n} \\
& =(q-1) \int_{0}^{1}\left|s w(x)+(1-s) u_{n}(x)\right|^{p-2}\left(w-u_{n}\right) d s \\
& =\int_{0}^{1} \frac{d}{d s}\left(\left|s w+(1-s) u_{n}\right|^{p-2}\left(s w+(1-s) u_{n}\right)\right) d s \\
& =w^{p-1}-\left|u_{n}\right|^{p-2} u_{n}
\end{aligned}
$$

and hence from $(*)$

$$
\begin{aligned}
- & \operatorname{div}\left(|\nabla w|^{p-2} \nabla w\right)-\left(-\operatorname{div}\left(\left|\nabla u_{n}\right|^{p-2} \nabla u_{n}\right)\right) \\
+ & \left(V \phi_{n}(x)-Q_{n} \psi_{n}\right) z_{n} \\
= & -\operatorname{div}|\nabla w|^{p-2}+V|w|^{p-2} w-Q_{n} w^{q-1} \\
& -\left[-\operatorname{div}|\nabla u|^{p-2} \nabla u+V|\nabla u|^{p-2} \nabla u-Q_{n}\left|u_{n}\right|^{q-2} u_{n}\right] \\
= & -\operatorname{div}|\nabla w|^{p-2}+V|w|^{p-2} w-Q_{n} w^{q-1} \\
\geqslant & -\operatorname{div}|\nabla w|^{p-2}+\delta_{\epsilon / 2} w^{q-1}=0 .
\end{aligned}
$$

Note that $V \phi_{n}(x)-Q_{n} \varphi_{n} \geqslant 0$ in $\Omega \backslash \overline{B_{\epsilon / 2}(0)}$ when $n \geqslant N_{\epsilon / 2}$. Due to the continuity of $u_{n}$ and the fact that $w_{n}(x) \rightarrow \infty$ as $x \rightarrow \partial B_{\epsilon / 2}(0)$, there is $r \in(\epsilon / 2, \epsilon)$ such that $z_{n} \geqslant 0$ on $\partial B_{r}(0)$. Moreover, $z_{n} \geqslant 0$ on $\partial \Omega$. If $\Omega$ is bounded, the maximum principle says that $z_{n} \geqslant 0$ in $\Omega \backslash B_{r}(0)$ (see $[20,21]$ ). If $\Omega$ is unbounded, by virtue of $w(x)$ tending to 0 as $|x| \rightarrow \infty$ by construction, thus for any $\gamma>0$, we may pick $\widetilde{R}>0$ such that $z_{n} \geqslant-\gamma$ in $\Omega \backslash B_{\widetilde{R}}(0)$. Moreover, applying regularity theory to $u_{n} \in W_{0}^{1, p}(\Omega)$, we can get $u_{n}(x) \rightarrow 0$ as $|x| \rightarrow \infty$. Now the same maximum principle is applied on $\Omega \cap\left(B_{\widetilde{R}} \backslash \overline{B_{r}(0)}\right)$, which implies that $z_{n} \geqslant-\gamma$ in all of $\Omega \backslash B_{r}(0)$. Letting $\gamma \rightarrow 0$, we obtain $z_{n}>0$ again. By analogy we obtain $u_{n} \geqslant-w$ (take $\left.z_{n}:=w+u_{n}\right)$; hence

$$
\left|z_{n}\right| \geqslant w \quad \text { in } \Omega \backslash B_{\epsilon}(0), \forall n \geqslant N_{\epsilon / 2} .
$$

Hence (a) follows from above arguments with the fact that $w$ is continuous in $\Omega \backslash B_{\epsilon}(0)$.

Next, the hypotheses in (b) imply that there is $\delta>0$ such that $Q_{n} \leqslant-\delta$ on $\Omega \backslash B_{1 / n}(0)$ for each $n$ large enough. Let $w_{n}$ be a positive solution to

$$
\begin{aligned}
-\operatorname{div}\left(|\nabla u|^{p-2} \nabla u\right) & =-\delta|u|^{q-2} u, \quad x \in \mathbb{R}^{n} \backslash B_{1 / n}(0) \\
\lim _{|x| \rightarrow 1 / n} w_{n}(x) & =+\infty, \quad \lim _{|x| \rightarrow \infty} w_{n}(x)=0 .
\end{aligned}
$$


Then the sequence $w_{n}$ is monotone decreasing, by using the maximum principle to $w_{n} \geqslant w_{n+1}$ on $\partial B_{1 / n}(0)$ for every $n \in \mathbb{N}$. Therefore, $w_{n}$ converges locally and uniformly to a nonnegative solution $w$ to (37) on $\mathbb{R}^{n} \backslash\{0\}$. It follows from our hypotheses on $N$ and $p$ that $w$ is an entire solution to (37) by applying the argument as in [24]. And then, due to [25], $w \equiv 0$. For another, the function $w_{n}$ dominates the solution $u_{n}$ on $\bar{\Omega} \backslash B_{r}(0)$ for some $r \in(\epsilon / 2, \epsilon)$, as seen in the proof of (a). Thus, $u_{n}$ also converges to 0 locally and uniformly in $\Omega \backslash B_{r}(0)$; that is, $\lim _{n \rightarrow \infty}\left|u_{n}\right|_{s, \Omega \backslash B_{r}(0)}=0$.

For $(c)$, we first consider the case $s(\geqslant 1) \in(N(q-p) / p, q]$. By interpolation inequality, we have the following estimate for solution $u_{n}$ :

$$
\begin{aligned}
\left\|u_{n}\right\|^{p} & =\int_{\Omega} Q_{n}\left|u_{n}\right|^{q} d x \leqslant c_{1}\left|u_{n}\right|_{q}^{q} \leqslant c_{1}\left|u_{n}\right|_{s}^{q \theta}\left|u_{n}\right|_{p^{*}}^{q(1-\theta)} \\
& \leqslant c_{2}\left|u_{n}\right|_{s}^{q \theta}\left\|u_{n}\right\|^{q(1-\theta)} .
\end{aligned}
$$

Here $c_{1}, c_{2}$ are independent of $n$, and $\theta$ satisfies that

$$
\frac{1}{q}=\frac{\theta}{s}+\frac{1-\theta}{p^{*}}
$$

According to Lemma 2, it suffices to impose that $q(1-\theta)<p$ or equivalent $s>N(q-p) / p$. This and (a) prove the case $s \in$ $(N(q-p) / p, q]$. And then, (38) and (a) yield $\left|u_{n}\right|_{q, B_{\epsilon}(0)} \rightarrow \infty$; hence $\left|u_{n}\right|_{s, B_{\epsilon}(0)} \rightarrow \infty$ for every $s \in(q, \infty]$ as $n \rightarrow \infty$. Using (a) again we get (30).

Note that (38) implies (30) for $s=N(q-p) / p$, so case (d) is easily followed.

\section{Concentration at Several Points}

Now we assume that the function $Q_{n}$ is positive in a neighbourhood of two distinct points $x_{1}, x_{2} \in \Omega$ (indeed, the following argument is also valid for any finite number of points in $\Omega$ ). More precisely, we assume.

$(* *) Q_{n}>0$ in a neighbourhood of $\left\{x_{1}\right\} \cup\left\{x_{2}\right\}$, and there exists a constant $C$ such that $\left|Q_{n}\right|_{L^{\infty}(\Omega)} \leqslant C$ for all $n$. Moreover, for each $\epsilon>0$ there exist constants $\delta_{\epsilon}>0$ and $N_{\epsilon}$ such that $Q_{n} \leqslant-\delta_{\epsilon}$ whenever $x \notin B_{\epsilon}\left(x_{1}\right) \cup B_{\epsilon}\left(x_{2}\right)$ and $n \geqslant N_{\epsilon}$.

As in Section 2, we put $J_{n}(u)=\int_{\Omega} Q_{n}|u|^{q} d x$ :

$$
s_{n}:=\inf _{J_{n}(u)} \frac{\|u\|^{p}}{J_{n}(u)^{p / q}} \equiv \inf _{J_{n}(u)} \frac{\int_{\Omega}\left(|\nabla u|^{p}+V|u|^{p}\right) d x}{\left(\int_{\Omega} Q_{n}|u|^{q} d x\right)^{p / q}} .
$$

Theorem 6. Suppose $Q_{n}$ satisfies (**) and $q \in\left(p, p^{*}\right)$, and $u_{n}$ is a ground state solution to (1). Then, for $n$ large, $u_{n}$ concentrates at $x_{1}$ or $x_{2}$. More precisely, for each $\epsilon>0$ we have by passing to a subsequence

$$
\begin{aligned}
& \lim _{n \rightarrow \infty} \frac{\int_{\Omega \backslash B_{\epsilon}\left(x_{j}\right)}\left(|\nabla u|^{p}+V u_{n}^{p}\right) d x}{\int_{\Omega}\left(|\nabla u|^{p}+V u_{n}^{p}\right) d x}=0, \\
& \lim _{n \rightarrow \infty} \frac{\int_{\Omega \backslash B_{\epsilon}\left(x_{j}\right)} Q_{n} u_{n}^{p} d x}{\int_{\Omega} Q_{n} u_{n}^{p} d x}=0
\end{aligned}
$$

for $j=1$ or 2 (but not for $j=1$ and 2 ).
Remark 7. Note that, in view of the obvious modification of Theorem 4 , the limits in (41) are 0 if $\Omega \backslash B_{\epsilon}\left(x_{j}\right)$ is replaced by $\Omega \backslash B_{\epsilon}\left(x_{1}\right) \cup B_{\epsilon}\left(x_{2}\right)$. So if $j=1$ in (41), then concentration occurs at $x_{1}$ and if $j=2$, it occurs at $x_{2}$.

Proof. As in [19], we may assume that $J_{n}\left(u_{n}\right)=$ $\int_{\Omega} Q_{n}\left|u_{n}\right|^{p} d x=1$ by renormalizing $\left(u_{n}\right.$ may not be a solution to (1), but we still have $\left.s_{n}:=\left\|u_{n}\right\|^{p} / J_{n}\left(u_{n}\right)^{p / q}\right)$. Let $\xi_{j} \in C_{0}^{\infty}(\Omega,[0,1])$ be a function such that $\xi_{j}=1$ on $B_{\epsilon / 2}\left(x_{j}\right)$ and $\xi_{j}=0$ on $\Omega \backslash B_{\epsilon}\left(x_{j}\right), j=1,2$, where $\epsilon$ is so small that $\overline{B_{\epsilon}\left(x_{j}\right)} \subset \Omega$ and $\overline{B_{\epsilon}\left(x_{1}\right)} \cap \overline{B_{\epsilon}\left(x_{2}\right)}=\emptyset$. Set $v_{n}:=\xi_{1} u_{n}, w_{n}:=\xi_{2} u_{n}$, and $z_{n}:=u_{n}-v_{n}-w_{n}$. Since supp $z_{n} \subset \Omega \backslash\left(B_{\epsilon / 2}\left(x_{1}\right) \cup B_{\epsilon / 2}\left(x_{2}\right)\right)$ and the conclusion of Theorem 4 remains valid after a modification, we have

$$
\begin{aligned}
\left\|u_{n}\right\|^{p}= & \int_{\Omega}\left(\left|\nabla u_{n}\right|^{p}+V u_{n}^{p}\right) d x \\
= & \left(\int_{\Omega}\left(\left|\nabla v_{n}\right|^{p}+V u_{n}^{p}\right) d x\right. \\
& \left.\quad+\int_{\Omega}\left(\left|\nabla w_{n}\right|^{p}+V w_{n}^{p}\right) d x\right)(1+o(1)) \\
= & \left(\left\|v_{n}\right\|^{p}+\left\|w_{n}\right\|^{p}\right)(1+o(1)), \\
1=J_{n}\left(u_{n}\right)= & \int_{\Omega} Q_{n}\left|u_{n}\right|^{q} d x \\
= & \int_{\Omega} Q_{n}\left|v_{n}\right|^{q} d x+\int_{\Omega} Q_{n}\left|w_{n}\right|^{q} d x+o(1) \\
= & J_{n}\left(v_{n}\right)+J_{n}\left(w_{n}\right)+o(1) .
\end{aligned}
$$

First, we assume that $\limsup _{n \rightarrow \infty} J_{n}\left(v_{n}\right) \geqslant 0$ and $\limsup _{n \rightarrow \infty} J_{n}\left(w_{n}\right) \geqslant 0$. By passing to a subsequence, we may assume that $J_{n}\left(v_{n}\right) \rightarrow c_{0} \in[0,1]$ and $J_{n}\left(w_{n}\right) \rightarrow 1-c_{0} \in$ $[0,1]$. If $c_{0} \in(0,1)$, recalling that $q>p$, we get a contradiction from the following inequality:

$$
\begin{aligned}
s_{n} & =\frac{\left\|u_{n}\right\|^{p}}{J_{n}\left(u_{n}\right)^{p / q}}=\frac{\left(\left\|v_{n}\right\|^{p}+\left\|w_{n}\right\|^{p}\right)(1+o(1))}{\left(J_{n}\left(v_{n}\right)+J_{n}\left(w_{n}\right)+o(1)\right)^{p / q}} \\
& >\frac{\left\|v_{n}\right\|^{p}+\left\|w_{n}\right\|^{p}}{J_{n}\left(v_{n}\right)^{p / q}+J_{n}\left(w_{n}\right)^{p / q}} \\
& \geqslant \min \left\{\frac{\left\|v_{n}\right\|^{p}}{J_{n}\left(v_{n}\right)^{p / q}}, \frac{\left\|w_{n}\right\|^{p}}{J_{n}\left(w_{n}\right)^{p / q}}\right\} \geqslant s_{n} .
\end{aligned}
$$

So $c_{0}=0$ or 1 . If $c_{0}=1$ (say), then the second limit in (41) is 0 for $j=1$ because $\operatorname{supp} v_{n} \subset B_{\epsilon}\left(x_{1}\right)$. The first limit is 0 as well, since $\left\|w_{n}\right\|^{p} /\left\|v_{n}\right\|^{p}$ is otherwise bounded away from 0 for large $n$, and we obtain a contradiction again from

$$
s_{n}=\frac{\left(\left\|v_{n}\right\|^{p}+\left\|w_{n}\right\|^{p}\right)(1+o(1))}{\left(J_{n}\left(v_{n}\right)+J_{n}\left(w_{n}\right)+o(1)\right)^{p / q}}>\frac{\left\|v_{n}\right\|^{p}}{J_{n}\left(v_{n}\right)^{p / q}} \geqslant s_{n} .
$$

Finally, suppose $\limsup _{n \rightarrow \infty} J_{n}\left(w_{n}\right)<0$ (the case $\lim \sup _{n \rightarrow \infty} J_{n}\left(v_{n}\right)<0$ is of course analogous); it passes to 
a subsequence $J_{n}\left(w_{n}\right) \leqslant-\eta$ for some $\eta>0$ when $n$ is large enough. Then a contradiction (44) holds for such $n$ because $J_{n}\left(v_{n}\right)>J_{n}\left(v_{n}\right)+J_{n}\left(w_{n}\right)+o(1)$.

\section{Conflict of Interests}

The author declares that there is no conflict of interests regarding the publication of this paper.

\section{Acknowledgments}

The author is greatly indebted to Professor A. Szulkin for providing the report about the concentration for the case $p=2$, which inspires them to consider concentration for the p-Laplacian equation. This paper is partially supported by the Science Foundation of Fujian Province (2012J05002), Post-Doctor Foundation of China Grant (2011M501074), and the Innovation Foundation of Fujian Normal University (IRTL1206).

\section{References}

[1] A. V. Buryak, P. di Trapani, D. V. Skryabin, and S. Trillo, "Optical solitons due to quadratic nonlinearities: from basic physics to futuristic applications," Physics Reports, vol. 370, no. 2, pp. 63$235,2002$.

[2] J. Byeon and Z. Q. Wang, "Standing waves with a critical frequency for nonlinear Schrödinger equations," Archive for Rational Mechanics and Analysis, vol. 165, no. 4, pp. 295-316, 2002.

[3] J. Byeon and Z. Q. Wang, "Standing waves with a critical frequency for nonlinear Schrödinger equations," Calculus of Variations and Partial Differential Equations, vol. 18, no. 2, pp. 207-219, 2003.

[4] J. Byeon and L. Jeanjean, "Standing waves for nonlinear Schrödinger equations with a general nonlinearity," Archive for Rational Mechanics and Analysis, vol. 185, no. 2, pp. 185-200, 2007.

[5] A. Ambrosetti, D. Arcoya, and J. L. Gámez, "Asymmetric bound states of differential equations in nonlinear optics," Rendiconti del Seminario Matematico della Università di Padova, vol. 100, pp. 231-247, 1998.

[6] D. Bonheure, J. M. Gomes, and P. Habets, "Multiple positive solutions of superlinear elliptic problems with sign-changing weight," Journal of Differential Equations, vol. 214, no. 1, pp. 3664, 2005.

[7] P. M. Girão and J. M. Gomes, "Multibump nodal solutions for an indefinite superlinear elliptic problem," Journal of Differential Equations, vol. 247, no. 4, pp. 1001-1012, 2009.

[8] W. Y. Ding and W. M. Ni, "On the existence of positive entire solutions of a semilinear elliptic equation," Archive for Rational Mechanics and Analysis, vol. 91, no. 4, pp. 283-308, 1986.

[9] S. Terracini, "On positive entire solutions to a class of equations with a singular coefficient and critical exponent," Advances in Differential Equations, vol. 1, no. 2, pp. 241-264, 1996.

[10] A. Ambrosetti and Z. Q. Wang, "Nonlinear Schrödinger equations with vanishing and decaying potentials," Differential and Integral Equations, vol. 18, no. 12, pp. 1321-1332, 2005.
[11] A. Lakmeche and A. Hammoudi, "Multiple positive solutions of the one-dimensional p-Laplacian," Journal of Mathematical Analysis and Applications, vol. 317, no. 1, pp. 43-49, 2006.

[12] J. B. Su, Z. Q. Wang, and M. Willem, "Weighted Sobolev embedding with unbounded and decaying radial potentials," Journal of Differential Equations, vol. 238, no. 1, pp. 201-219, 2007.

[13] D. Motreanu, V. V. Motreanu, and N. S. Papageorgiou, "A multiplicity theorem for problems with the $p$-Laplacian," Nonlinear Analysis: Theory, Methods \& Applications, vol. 68, no. 4, pp. 1016-1027, 2008.

[14] J. H. Zhao and P. H. Zhao, "Existence of infinitely many weak solutions for the $p$-Laplacian with nonlinear boundary conditions," Nonlinear Analysis: Theory, Methods \& Applications, vol. 69, no. 4, pp. 1343-1355, 2008.

[15] D. G. de Figueiredo, J. P. Gossez, and P. Ubilla, "Local "superlinearity" and "sublinearity" for the p-Laplacian," Journal of Functional Analysis, vol. 257, no. 3, pp. 721-752, 2009.

[16] F. Torre and B. Ruf, "Multiplicity of solutions for a superlinear p-Laplacian equation," Nonlinear Analysis: Theory, Methods \& Applications, vol. 73, no. 7, pp. 2132-2147, 2010.

[17] L. Iturriaga, S. Lorca, and E. Massa, "Positive solutions for the p-Laplacian involving critical and supercritical nonlinearities with zeros," Annales de l'Institut Henri Poincare C: Non Linear Analysis, vol. 27, no. 2, pp. 763-771, 2010.

[18] S. B. Liu, "On ground states of superlinear $p$-Laplacian equations in $\mathbb{R}^{N}$," Journal of Mathematical Analysis and Applications, vol. 361, no. 1, pp. 48-58, 2010.

[19] N. Ackermann and A. Szulkin, "A concentration phenomenon for semilinear elliptic equations," Archive for Rational Mechanics and Analysis, vol. 207, no. 3, pp. 1075-1089, 2013.

[20] J. García-Melián and J. Sabina de Lis, "Maximum and comparison principles for operators involving the $p$-Laplacian," Journal of Mathematical Analysis and Applications, vol. 218, no. 1, pp. 49-65, 1998.

[21] M. Montenegro, "Strong maximum principles for supersolutions of quasilinear elliptic equations," Nonlinear Analysis: Theory, Methods \& Applications, vol. 37, no. 4, pp. 431-448, 1999.

[22] G. Díaz and R. Letelier, "Explosive solutions of quasilinear elliptic equations: existence and uniqueness," Nonlinear Analysis: Theory, Methods \& Applications, vol. 20, no. 2, pp. 97-125, 1994.

[23] J. García-Melián, "Nondegeneracy and uniqueness for boundary blow-up elliptic problems," Journal of Differential Equations, vol. 223, no. 1, pp. 208-227, 2006.

[24] H. Brézis and L. Véron, "Removable singularities for some nonlinear elliptic equations," Archive for Rational Mechanics and Analysis, vol. 75, no. 1, pp. 1-6, 1980.

[25] C. Bandle and M. Marcus, "“Large” solutions of semilinear elliptic equations: existence, uniqueness and asymptotic behaviour," Journal d'Analyse Mathématique, vol. 58, no. 1, pp. 9-24, 1992. 


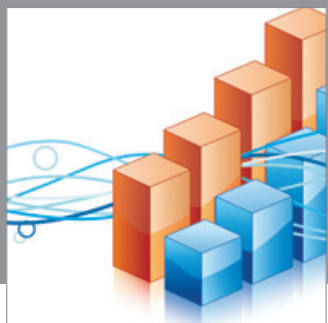

Advances in

Operations Research

mansans

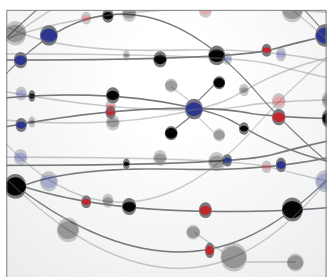

The Scientific World Journal
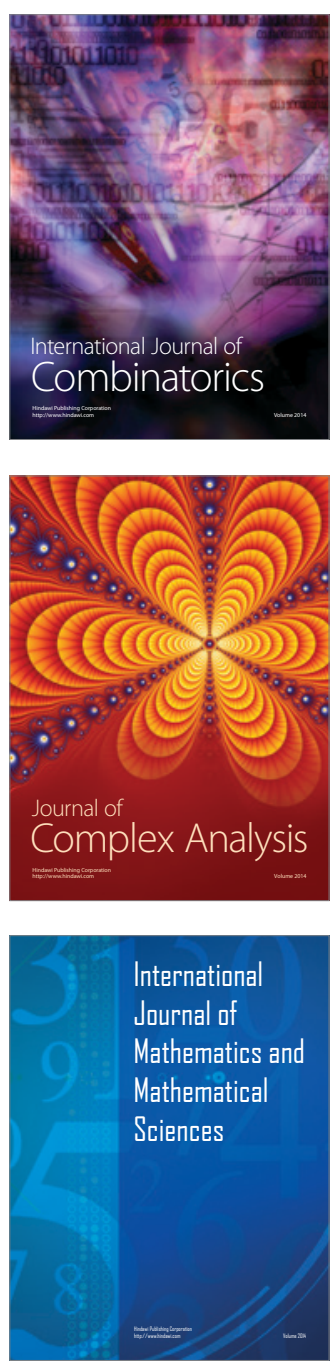
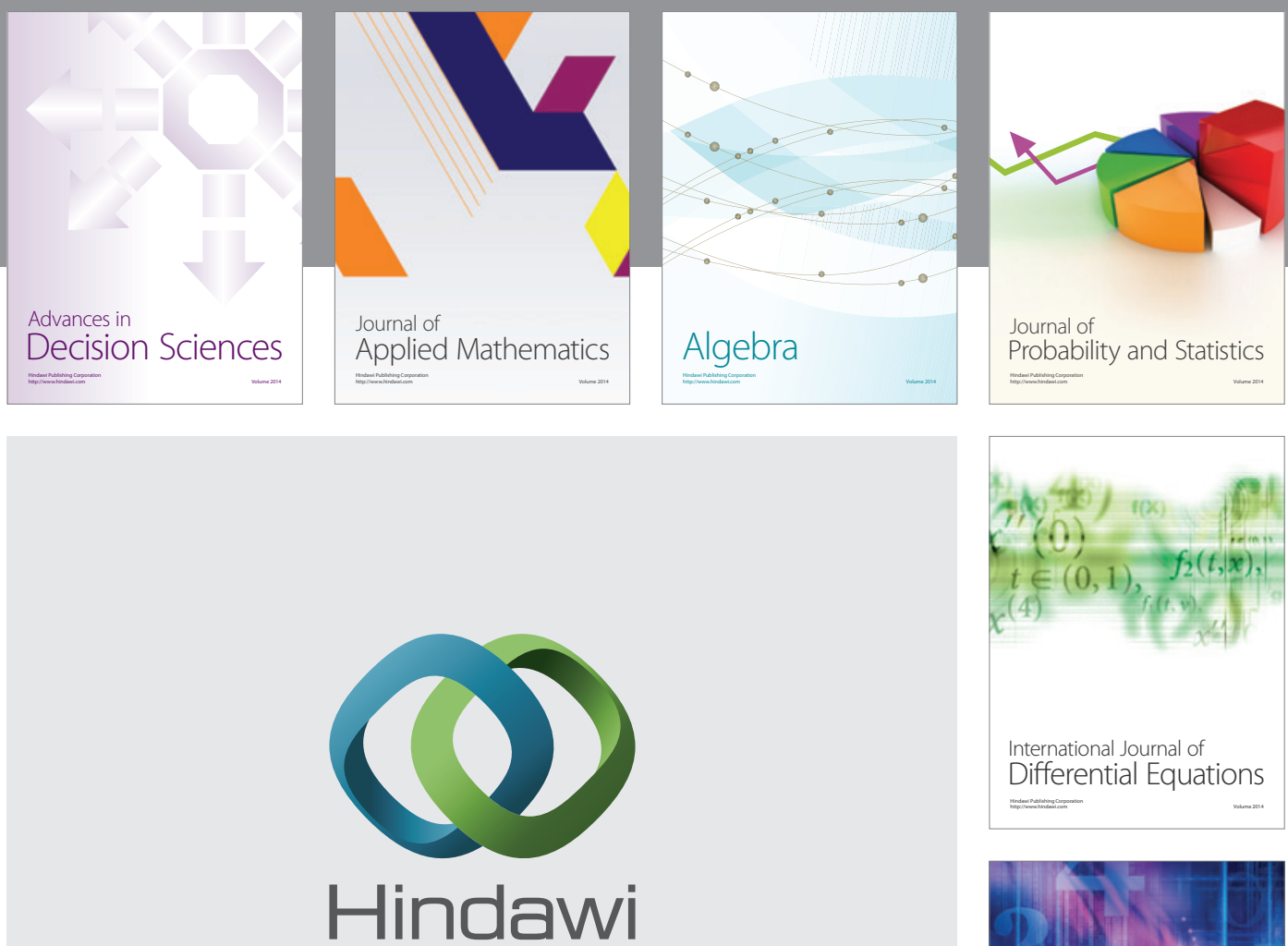

Submit your manuscripts at http://www.hindawi.com
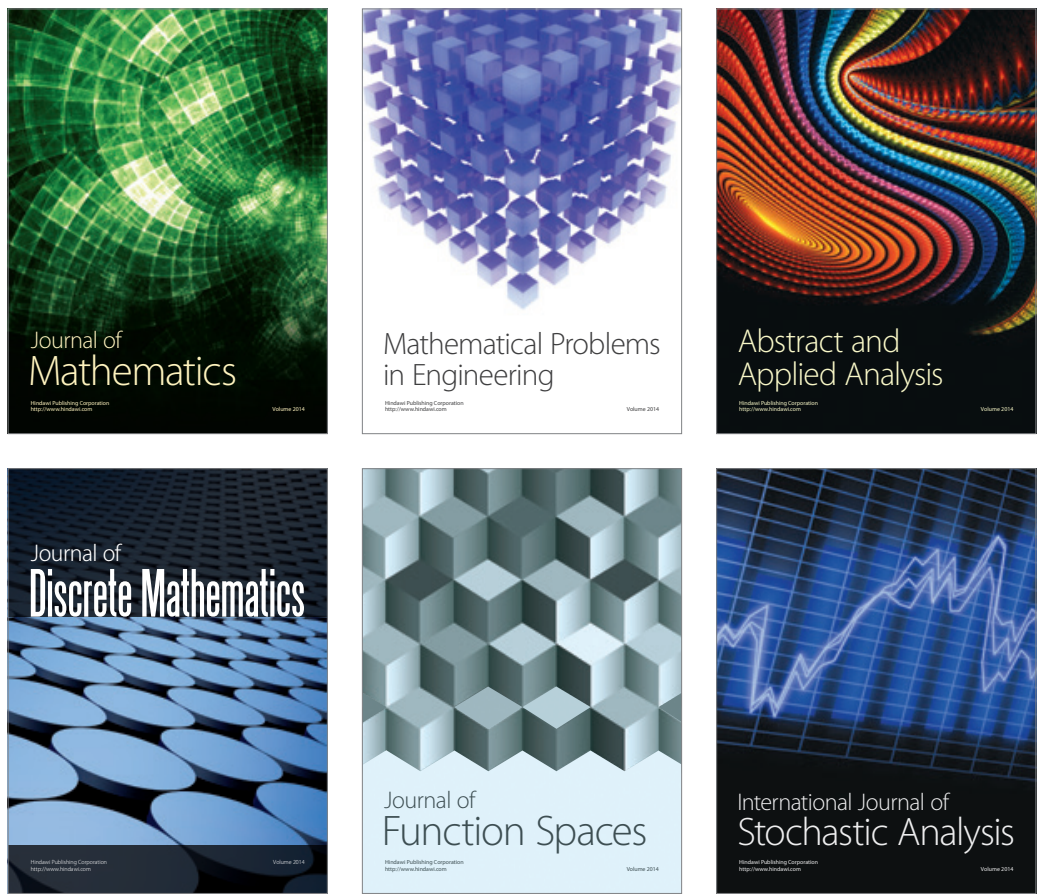

Journal of

Function Spaces

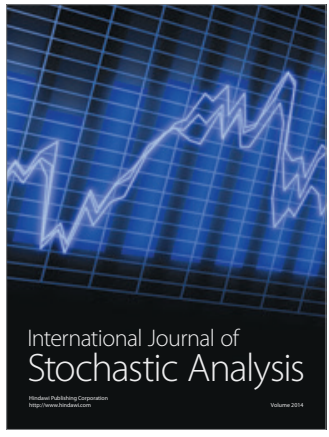

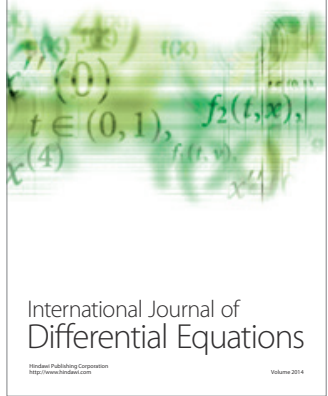
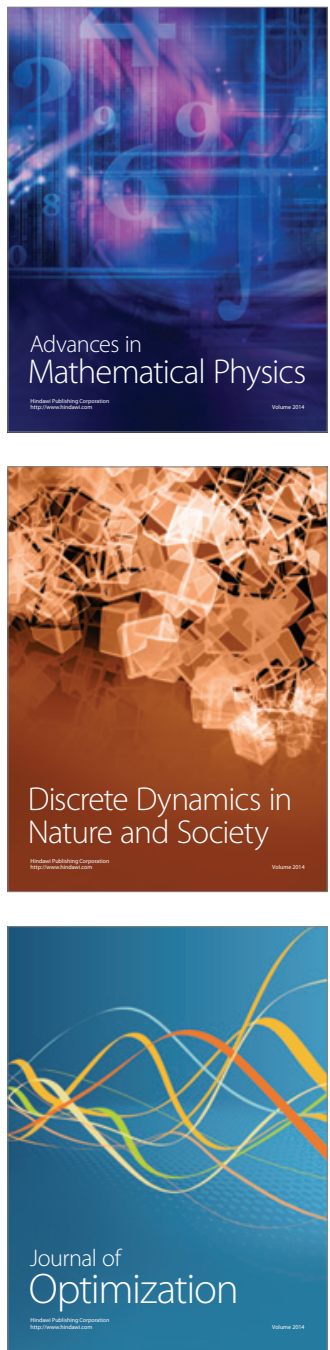